\title{
Editorial
}

\section{Time-Dependent Billiards}

\author{
Alexander Loskutov ${ }^{1,2}$ and Edson D. Leonel ${ }^{2}$ \\ ${ }^{1}$ Physics Faculty, Moscow State University, Moscow 119992, Russia \\ ${ }^{2}$ Departamento de Estatística, Matemática Aplicada e Computação, Universidade Estadual Paulista, \\ Avenida 24A 1515, 13506-700 Rio Claro, SP, Brazil
}

Correspondence should be addressed to Edson D. Leonel, edleonel@rc.unesp.br

Received 24 August 2009; Accepted 24 August 2009

Copyright (C 2009 A. Loskutov and E. D. Leonel. This is an open access article distributed under the Creative Commons Attribution License, which permits unrestricted use, distribution, and reproduction in any medium, provided the original work is properly cited.

\section{Introduction}

Dynamical systems of a billiard type are a fundamental notion relevant for the understanding of numerous phenomena observed in statistical mechanics, Hamiltonian dynamics, nonlinear physics, and others. By means of billiard models, the principal ideas as Boltzmann ergodic hypothesis, related to the foundation of statistical physics, and deterministic diffusion gained more deep insight. However, more sophisticated and realistic results may be obtained if billiards are considered with time-dependent boundaries. Such kind of billiard systems represent a natural generalization of mathematical billiards and more adequately reflects the observed physical phenomena. This Focus Issue presents recent progress in this area with contributions on new ways and specific model studies.

\section{Context}

In the last four decades of the twentieth century, mathematical billiards and related fields became one of the most active research areas in statistical mechanics and theory of dynamical systems. However, the history of billiard problems started in 1927 with a remarkable paper by Birkhoff [1], who considered a problem concerning the free motion of a point particle (billiard ball) in some bounded manifold. A more complete consideration of the problems related to the dynamics of mass points in a bounded region was given by Krylov [2]. However, the contemporary investigations of billiards were initiated by Sinai [3] and some time later by Bunimovich [4] (see also a classical paper on the Lorenz gas [5]), who made the rigorous analysis of the billiard dynamics. A great progress of billiard's dynamics was achieved in a solution, in a certain sense, of the known Boltzmann ergodic hypothesis. 
A billiard dynamical system is generated by the free motion of a point mass particle (billiard ball) in some region with a piecewise-smooth boundary and the condition of the elastic collision from this boundary. If the boundary in the collision point is smooth, then the billiard ball reflects from it in such a way that the velocity tangent component remains constant, and the normal component changes its sign. If the ball hits a corner of the billiard table, then its dynamics is not determined.

Nowadays, the main focus in the study of billiard problems is moving from the investigations of billiards with the fixed boundary to time-dependent billiards. Indeed, the Lorentz gas has been proposed for the description of the motion of electrons between heavy ions in the lattice of metals. In the reality, however, ions should weakly oscillate near their equilibrium state. Moreover, some important problems of mathematical physics can be described by nonstationary billiard models (see [6]).

For the most part, investigations of classical time-dependent billiards concerned two main questions: descriptions of their statistical properties and the study of trajectories for which the particle velocity grows indefinitely. This problem is related to the unbounded increase of energy in periodically forced Hamiltonian systems and known as Fermi acceleration [7]. In his paper, Fermi supposed that in a typical environment, the probability of a head-on collision is greater than the probability of a head-tail collision, so particles would, on average, be accelerated. Later many approaches regarding the description of such a phenomenon were introduced for both the continuous and discrete time models (see [6, 8$11]$ and references cited therein).

It is obvious that in this context dynamical properties of billiards play a principal role: if it is chaotic, then the boundary perturbation may lead to the particle acceleration. In papers $[12,13]$, on the basis of these ideas, the following conjecture, known in literature as Loskutov-Ryabov-Akinshin- (LRA-) conjecture (see, e.g., [14-16]) has been advanced: Fermi acceleration will be observed in time-dependent billiards if in the autonomous case these billiards possess chaotic properties. This conjecture has been confirmed for Bunimovich stadium [17] and for annular billiards [18]. Lately, using the theory of dynamical systems, it was proved that the Fermi acceleration in some nonautonomous billiard-like systems should be observed $[19,20]$. Recently this phenomenon has been detected for driven elliptical billiard [9]. Finally, applying thermodynamic methods, it was shown [21] that Fermi acceleration is inherent in the Lorentz gas of a quite general configuration.

The next physical generalization of billiard ideas is to consider the corresponding dynamical systems with inelastic collisions. These models admits more realistic analysis of some natural phenomena. For example, they allow us to study the presence and lack of Fermi acceleration in certain billiard-like models.

\section{This Focus Issue}

The contributions to this focus issue can be grouped into three main parts:

(i) Hamiltonian dynamics and related fields;

(ii) dynamics of time-dependent billiard-like models;

(iii) applications.

The first part on Hamiltonian systems addresses problems such as integrability and nonintegrability, scaling results for the phase transition, properties of chaotic regimes in the 
region below the first invariant spanning curve, chaotic properties and structure of the phase space, and stability of periodic time-dependent systems.

The second part contains in-depth studies of specific models of time-dependent billiardlike models and nonautonomous systems. Here, the following questions are considered: in what cases Fermi acceleration can be observed?, how one can describe the scaling properties of billiard-like systems?, does adding the inelasticity lead to suppression of Fermi acceleration?

The third part on Applications contains contributions on several generalizations related to accelerator models, analysis of certain mechanical billiard-like systems, and some quantum abstractions.

We do hope that the presented papers will be interested in a wide audience of readers.

\section{Acknowledgments}

Alexander Loskutov gratefully acknowledges FAPESP and Departamento de Estatística, Matemática Aplicada e Computação (Brazil) for the summer grant during his stay in the Universidade Estadual Paulista, Rio Claro, Brazil. E. D. Leonel acknowledges the financial support from CNPq, FAPESP, CAPES, FUNDUNESP and Pró Reitoria de Pesquisa (PROPe, UNESP) and Pró Reitoria de Pós-Graduação (PROPG, UNESP). They are grateful to Professors Makoto Yoshida (UNESP, Brazil), Jafferson Silva (UFMG, Brazil), and Leonid Bunimovich (Georgia Institute of Tecnhology, USA), and all the contributors of the past International Meeting "Billiards'09" (Águas de Lindóia, Brazil), as well as the authors for their papers sent to the present issue.

Alexander Loskutov Edson D. Leonel

\section{References}

[1] G. Birkhoff, Dynamical Systems, American Mathematical Society, Providence, RI, USA, 1927.

[2] N. S. Krylov, Works on the Foundations of Statistical Physics, Princeton University Press, Princeton, NJ, USA, 1979.

[3] Ya. G. Sinai, "Dynamical systems with elastic reflections," Russian Mathematical Surveys, vol. 25, no. 2, pp. 137-189, 1970.

[4] L. A. Bunimovich, "On the ergodic properties of certain billiards," Functional Analysis and Its Applications, vol. 8, pp. 73-74, 1974.

[5] L. A. Bunimovich and Ya. G. Sinai, "Statistical properties of Lorentz gas with periodic configuration of scatterers," Communications in Mathematical Physics, vol. 78, no. 4, pp. 479-497, 1981.

[6] A. Loskutov, "Dynamical chaos: systems of classical mechanics," Physics-Uspekhi, vol. 50, no. 9, pp. 939-964, 2007.

[7] E. Fermi, "On the origin of the cosmic radiation," Physical Review, vol. 75, no. 8, pp. 1169-1174, 1949.

[8] A. J. Lichtenberg and M. A. Lieberman, Regular and Stochastic Motion, Springer, New York, NY, USA, 1992.

[9] A. K. Karlis, P. K. Papachristou, F. K. Diakonos, V. Constantoudis, and P. Schmelcher, "Fermi acceleration in the randomized driven Lorentz gas and the Fermi-Ulam model," Physical Review E, vol. 76, no. 1, Article ID 016214, 2007.

[10] E. D. Leonel, "Breaking down the Fermi acceleration with inelastic collisions," Journal of Physics A, vol. 40, no. 50, pp. F1077-F1083, 2007.

[11] E. D. Leonel and M. R. Silva, "A bouncing ball model with two nonlinearities: a prototype for Fermi acceleration," Journal of Physics A, vol. 41, no. 1, Article ID 015104, 13 pages, 2008.

[12] A. Yu. Loskutov, A. B. Ryabov, and L. G. Akinshin, "Mechanism of Fermi acceleration in dispersing billiards with time-dependent boundaries," Journal of Experimental and Theoretical Physics, vol. 89, no. 5, pp. 966-974, 1999. 
[13] A. Loskutov, A. B. Ryabov, and L. G. Akinshin, "Properties of some chaotic billiards with timedependent boundaries," Journal of Physics A, vol. 33, no. 44, pp. 7973-7986, 2000.

[14] R. E. de Carvalho, F. C. de Souza, and E. D. Leonel, "Fermi acceleration on the annular billiard," Physical Review E, vol. 73, no. 6, Article ID 066229, 2006.

[15] A. L. P. Livorati, D. G. Ladeira, and E. D. Leonel, "Scaling investigation of Fermi acceleration on a dissipative bouncer model," Physical Review E, vol. 78, no. 5, Article ID 056205, 2008.

[16] F. Lenz, F. K. Diakonos, and P. Schmelcher, "Tunable fermi acceleration in the driven elliptical billiard," Physical Review Letters, vol. 100, no. 1, Article ID 014103, 2008.

[17] A. Loskutov and A. Ryabov, "Particle dynamics in time-dependent stadium-like billiards," Journal of Statistical Physics, vol. 108, no. 5-6, pp. 995-1014, 2002.

[18] R. E. de Carvalho, F. C. de Souza, and E. D. Leonel, "Fermi acceleration on the annular billiard: a simplified version," Journal of Physics A, vol. 39, no. 14, pp. 3561-3573, 2006.

[19] V. Gelfreich and D. Turaev, "Fermi acceleration in non-autonomous billiards," Journal of Physics A, vol. 41, no. 21, Article ID 212003, 6 pages, 2008.

[20] V. Gelfreich and D. Turaev, "Unbounded energy growth in Hamiltonian systems with a slowly varying parameter," Communications in Mathematical Physics, vol. 283, no. 3, pp. 769-794, 2008.

[21] A. Loskutov, O. Chichigina, and A. Ryabov, "Thermodynamics of dispersing billiards with timedependent boundaries," International Journal of Bifurcation and Chaos, vol. 18, no. 9, pp. 2863-2869, 2008. 


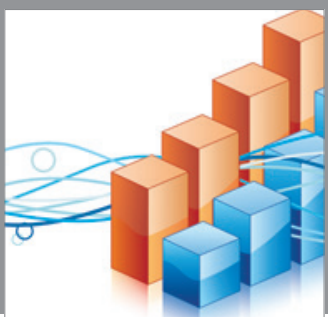

Advances in

Operations Research

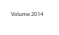

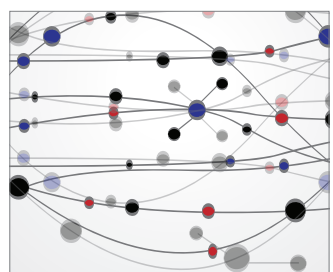

\section{The Scientific} World Journal
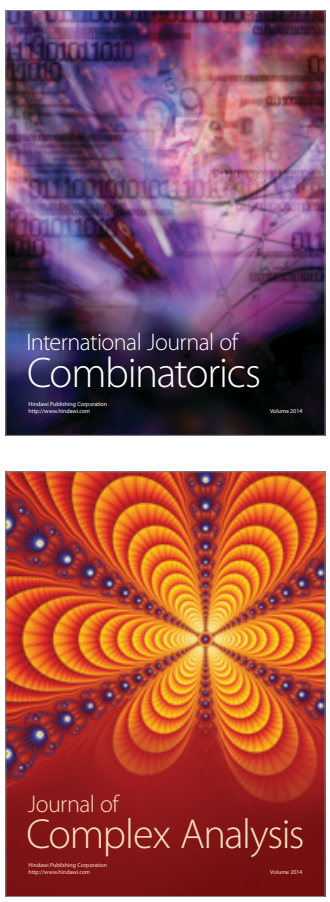

International Journal of

Mathematics and

Mathematical

Sciences
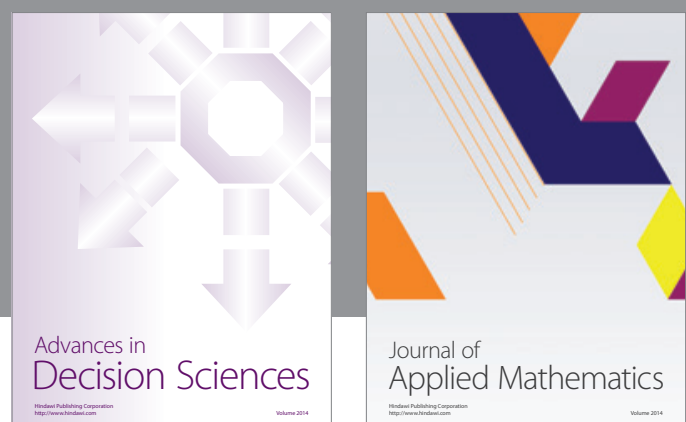

Journal of

Applied Mathematics
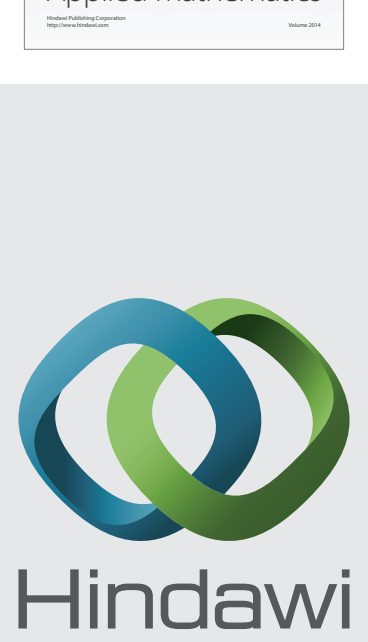

Submit your manuscripts at http://www.hindawi.com
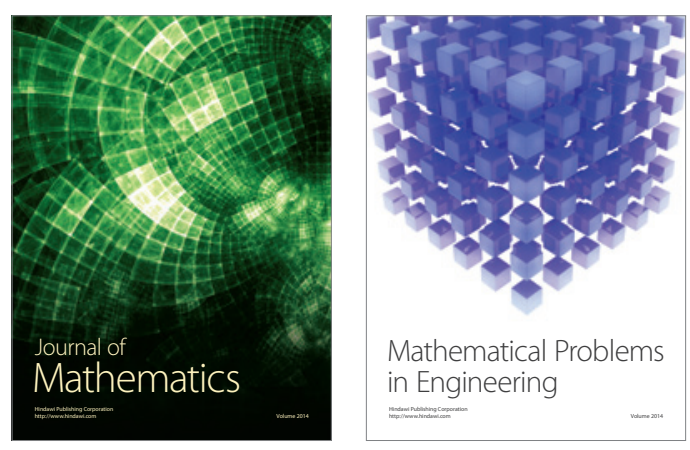

Mathematical Problems in Engineering
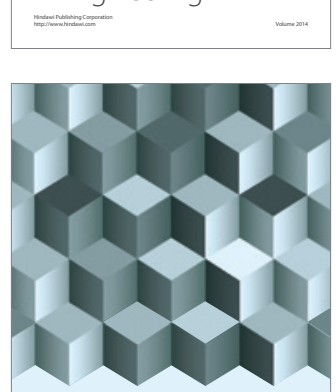

Journal of

Function Spaces
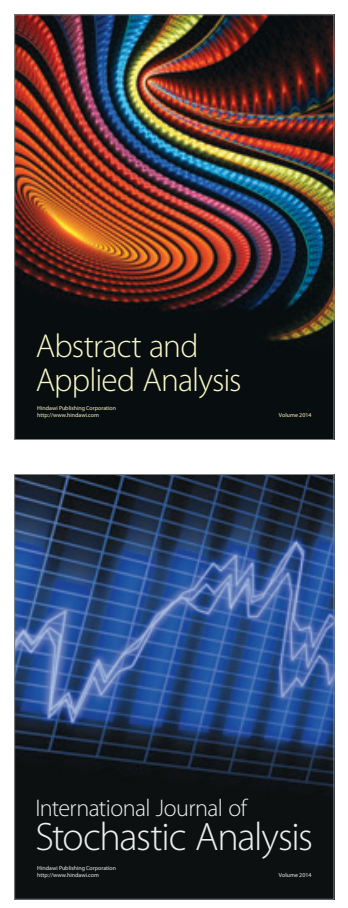

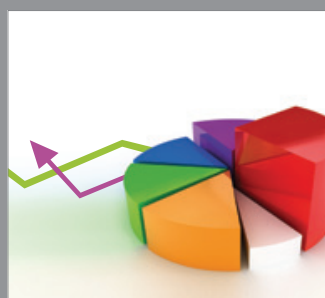

ournal of

Probability and Statistics

Promensencen
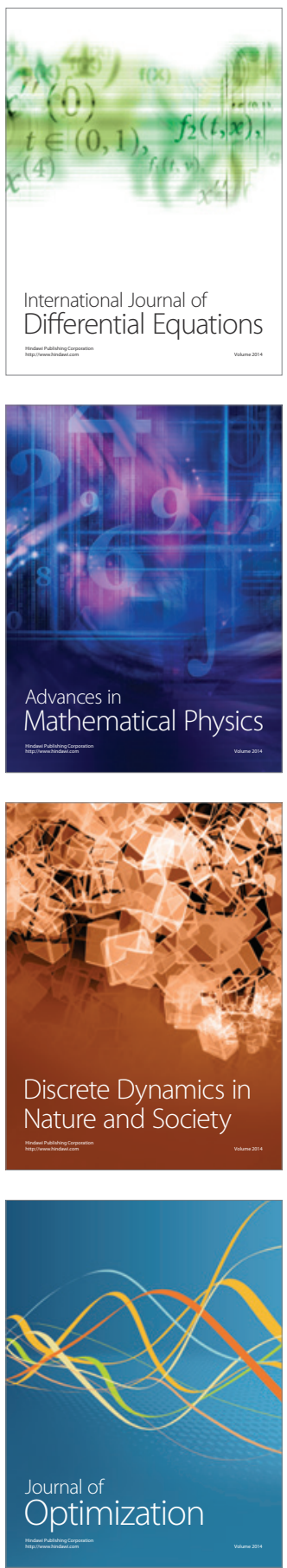